Proc. Indian Acad. Sci, (Math. Sci.), Vol, 89, Number 1, January 1980, pp. 25-33. (C) Printed in India.

\title{
Nonnegative integral solution of linear equations
}

\author{
S K SEN \\ Computer Centre, Indian Institute of Science, Bangalore 560012 \\ MS received 7 September 1978
}

\begin{abstract}
A method to obtain a nonnegative integral solution of a system of linear equations, if such a solution exists is given. Th.e mett.od writes linfar equations as an integer programming problem and then solves the problem using a ccmbination of artificial basis technique and a method of integer forms.
\end{abstract}

Keywords. All-integer programming; artificial basis technique; Gomory method of integer forms; linear programming; non-negative integral solution.

\section{Introduction}

Many problems like those of path-length, fixed-charge, batch-size, transportation and allocation, chemical reactor vessel (Gottfried and Weisman [7]), computer networking involve quantities which can be only nonnegative integers. Such a problem giving rise to linear equations needs nonnegative integral solution.

Hurt and Waid [8] propose a generalized inverse $A^{-}$which gives the general integral solution (all the integral solutions) to linear equations (also Ben-Israel and Greville [1]; Marcus and Minc [9]; Sen and Shamim [12]). There seems to be no easy way to seeve out nonnegative integral solvtions from the general form $\mathrm{x}=$ $A^{-} b+\left(I-A^{-} A\right)$ y where $\mathrm{y}$ and $I$ are arbitrary integral $n$-vector and $n \times n$ unit matrix, respectively. $A \mathrm{x}=\mathrm{b}$ are the equations where $A$ is an $m \times n$ integral matrix.

The method described here investigates equations $A \mathrm{x}=\mathrm{b}$, consistent or not, underdetermined or overdetermined as an all-integer programming (all-ip) problem and gives a nonnegative integral solution $x$ when it exists. To solve the all-ip problem the method involves a particular form of the artificial basis technique (Sen [11]; Chung [2]; Strum [13]) and the Gomory method of integer forms (Gomory [6]; Vajda [15]; Salkin [10]).

\section{The problem}

Obtain a nonnegative integral solution $\mathrm{x}$ of $A \mathrm{x}=\mathrm{b}$ (if it exists) where $A=\left(a_{i j}\right)$ is a given $m \times n$ integral matrix, $\mathrm{b}=\left(b_{i}\right)$ is a given non-negative integral $m$-vector and $\mathrm{x}=\left(x_{\xi}\right)$ is an $n$-vector. 
Note. There is no loss of generality in considering (i) $b \geqslant 0$ and (ii) $A$ and $b$ integral. For, if these are not then multiply the equations with negative $b_{i}$ by -1 and nonintegral equations by suitable scalars.

\section{Existence of a nonnegative integral solution}

$A \mathrm{x}=\mathrm{b}$ has a non-negative solution $\mathrm{x}$ if and only if $A^{t} \mathrm{y} \geqslant 0, \mathrm{~b}^{t} \mathrm{y}<0$ has no solution y. $t$ indisates the transpose (Vajda [14]; Farkas [3]).

Equivalently, $A \mathrm{x}=\mathrm{b}$ has no nonnegative solution $\mathrm{x}$ if and only if $A^{\mathrm{t}} \mathrm{y} \geqslant 0$, $\mathrm{b}^{t} \mathrm{y}<0$ has a solution $\mathrm{y}$.

Let $A$ and $\mathrm{b}$ be integral and $A \mathrm{x}=\mathrm{b}$ be consistent (i.e., $A A^{-} \mathrm{b}=\mathrm{b}$ ). Also, let $A \mathrm{x}=\mathrm{b}$ have a nonnegative solution. If $A$ is nonsingelar and its inverse is also integral then $A \mathrm{x}=\mathrm{b}$ has unique nonnegative integral solution $\mathrm{x}=A^{-1} \mathrm{~b}$. Further, if $\mathrm{x}=A^{-} \mathrm{b}$ is not integral then $A \mathrm{x}=\mathrm{b}$ has no integral solution (it has non-integral rational solutions though). $A^{-}$here is a (reflexive) generalized inverse that satisfles

$$
A A^{-} A=A, A^{-} A A^{-}=A^{-}, A^{-} A \text { and } A A^{-} \text {are integral. }
$$

These results are not of immediate use. However, the method tells if a nonnegative solution of $A \mathrm{x}=\mathrm{b}$ does not exist. In fact, the necessary and sufficient condition for $A \mathrm{x}=\mathrm{b}$ to have a nonnegative solution is the method producing one. Further, the sufficient condition that this solution is integral is the (Gomory) method giving one.

\section{The method}

The method consists of two parts.

Part 1 (Equivalent ip problem). Write (1) as an all-ip problem.

Part 2 (Gomory-artificial-basis technique). Solve this ip problem using Gomory method of integer forms in which a particular form of the artificial basis technique is embedded.

\section{(i) Equivalent ip problem}

Let $\mathrm{x}$ and $A$ be now extended $(n+m)$-vector and $m \times(n+m)$ matrix, respectively. Further, let the last $m$ columns of $A$ form an $m \times m$ unit matrix. The $i p$ problem equivalent to (1) is

Obtain $\mathrm{x}$ so that

Min $z=x_{n+1}+\ldots+x_{n+m}=0:$ Objective function

subject to

$A x=b$ : Constraints

$x \geqslant 0$ and integral : Nonnegativity and integrality conditions.

\section{(ii) Gomory-artificial-basis technique}

Step 1. Solve the ip problem as a linear programming $(l p)$ problem using the artificial basis technique in 'restricted tableau' (described later). If it is infeasible, 
so is the ip problem-terminate. If the optimal solution is all integer then the ip problem is solved-terminate. Otherwise go to step 2.

Step 2. Consider one of the variables which have a fraction* in their value in the optimal (simplex) restricted tableau.

(i) Let the row of such a variable be

$$
\begin{array}{lllll}
x & z_{1} & \ldots & z_{t} & z_{0}
\end{array}
$$

where $z_{0}$ is the present value of the variable $x$.

(ii) Write every $z_{i}$ as $L_{i}+f_{i}$, where $L_{i}=$ the largest integer contained in $z_{i}$ and hence $f_{i}$ is nonnegative. In particular, $f_{0}$ is positive.

(iii) Add to the (simplex) tableau the further row (Gomory constraints)

$$
s_{1}-f_{1} \ldots-f_{t}-f_{0} .
$$

(iv) Apply the Dual Simplex method (described later) on this tableau. This renders the new variable $s_{1}$ non-basic.

Note. The Simplex tableau is already dual feasible, since the final tableau of the artificial basis technique (Simplex method) is reached. So the Dual Simplex method has been used.

Step 3. If the result again contains a basic variable which is not an integer then continue introducing new variables, $s_{2}, \ldots$. The methcd terminates in a finite number of steps if the feasible region of the ip problem is bounded (st:fficient but not necessary).

\section{Artificial basis technique in restricted tableau}

Step 1. Set up the restricted Simplex tableau for (2), and write the coefficients (in parentheses) which $x_{j}$ have in the objective ft:ncticn and the last row, i.e.,

\begin{tabular}{|c|c|c|c|c|}
\hline & $(0)$ & $(0)$ & $(0)$ & \\
\hline & $x_{1}$ & $x_{j_{0}}$ & $x_{n}$ & $b$ \\
\hline (1) $x_{n+1}$ & $\begin{array}{c}a_{11} \\
:\end{array}$ & $a_{1 \%}$ & $a_{1 n}$ & $b_{1}$ \\
\hline (1) $x_{n-1}$ & $a_{b_{0} 1}$ & $a_{\boldsymbol{t}_{0} g_{0}}$ & $a_{\mathrm{in}^{n}}$ & $b_{t_{0}}$ \\
\hline (1) $x_{n+m}$ & $\begin{array}{c}a_{m 1} \\
d_{1}\end{array}$ & $\begin{array}{c}a_{m ! \cdot} \\
d .0\end{array}$ & $\begin{array}{l}a_{m n} \\
d_{n}\end{array}$ & $b_{m}$ \\
\hline
\end{tabular}
$d_{j}$-row using the checking rule (described later) as below

Step 2. (pivot selection). Let $d_{y_{0}}$ be positive. Consider then, for all positive $a_{i s_{0}}$, the ratios $b_{\mathbf{b}} / a_{i j_{0}}$ and take a smallest. If this is obtained for $i_{0}$ then call $p=a_{w_{0}}$

- It is generally assumed that convergence is speeded by choosing that cut which bites as deeply as possible. This is usually taken to mean the selection of the row that gives the largest fraction $\left(f_{0}\right)$. 
the pivot (marked with a plus). Go to Step 3. Otherwise the present tableau is final and it either indicates no solution of $A x=b$ or gives a nonnegative solution.

Step 3 (next-tableau computation). Having interchanged $x_{\nu_{0}}$ and $x_{m+t_{0}}$ obtain the next tableau as follows.

$$
\begin{aligned}
& \begin{array}{lllllll}
x_{1} & \ldots & x_{n+i_{0}} & \ldots & x_{n} & \mathrm{~b}
\end{array} \\
& x_{n+1} \quad-a_{1 j_{0}} / p \\
& \begin{array}{lllll}
x_{j} & a_{i_{0} 1} / p & 1 / p & a_{i_{\text {on }}} / p & b_{i_{0}} / p
\end{array} \\
& x_{n+m} \quad-a_{m j_{0}} / p \\
& -d_{s_{0}} / p
\end{aligned}
$$

The blank positions are flled in as follows :

$$
\begin{aligned}
& a_{i j} \leftarrow a_{i j}-a_{i_{0} j} a_{i j_{3}} / p \\
& d_{j} \leftarrow d_{i}-a_{i_{0} j} d_{i 0} / p .
\end{aligned}
$$

Note. ' $\leftarrow$ ' means ' is replaced by'.

(i) The foregoing two 'replacements' are actually identical when we consider the last row (i.e., $d_{j}$-row) as just another row like the rous of $\left(a_{i j}\right)$.

(ii) The right-hand side elements are the elements of the foregoing tableau throughout the computation.

Step 4 (termination condition). If the bottom row i.e. $d_{5}$-row excluding the last element is nonpositive, or if none of $x_{n+1}, \ldots, x_{n+m}$ occurs in the basis with a nonzero value then a nonnegative solution is reached. Otherwise go to step 2 .

6. The checking rule for a simplex tableau

Let the $l p$ problem be

Minimize $z=\mathrm{c}^{t} \mathrm{x}$ subject to $A \mathrm{x}=\mathrm{y}, \mathrm{x} \geqslant 0$

where

$$
\mathrm{c}=\left[\begin{array}{c}
c \\
\vdots \\
c_{n}
\end{array}\right], \mathrm{x}=\left[\begin{array}{c}
x_{1} \\
\vdots \\
x_{n}
\end{array}\right], y=\left[\begin{array}{c}
b_{1} \\
\vdots \\
b_{m}
\end{array}\right], A=\left[\begin{array}{ccc}
a_{-1} & \ldots & a_{: n} \\
\vdots & & \vdots \\
a_{m 1} & \ldots & a_{m n}
\end{array}\right] .
$$

We attach to all variables $x_{i}$ the coefficient which they have in the objective function. Let, for example, a current 'restricted tableau' be

$\begin{array}{llllll} & \left(c_{1}\right) & \left(c_{6}\right) & \left(c_{2}\right) & \left(c_{4}\right) & \\ & x_{1} & x_{6} & x_{2} & x_{4} & \\ \left(c_{3}\right) x_{3} & p_{11} & p_{12} & p_{13} & p_{14} & v_{1} \\ \left(c_{5}\right) x_{5} & p_{21} & p_{22} & p_{23} & p_{24} & v_{2} \\ & d_{1} & d_{2} & d_{3} & d_{4} & d_{5}\end{array}$


Then

$$
\begin{aligned}
& c_{3} p_{11}+c_{5} p_{21}-c_{1}=d_{1} \\
& c_{3} p_{12}+c_{5} p_{22}-c_{6}=d_{2} \\
& c_{3} p_{14}+c_{5} p_{24}-c_{2}=d_{3} \\
& c_{3} v_{1}+c_{5} v_{2}=d_{5} .
\end{aligned}
$$

Such a relationship holds in all tableaux. This relationship is referred to as the checking rule for a tableau. Satisfaction of this rule is necessary for a restricted tableau to be correct but it is not sufficient (i.e., the rule may be satisfied even if a computational mistake occurs).

\section{The dual simplex method}

When to use. Let an $l p$ problem be

Also, let

$$
\begin{array}{ll}
\text { Minimize } & z=c_{1} x_{1}+\cdots+c_{n} x_{n} \\
\text { subject to } & a_{11} x_{1}+\cdots+a_{1 n} x_{n}+a_{1 n+1} x_{n+1}=b_{1} \\
& \vdots \\
& a_{m 1} x_{1}+\cdots+a_{m n} x_{n}+a_{m n+1} x_{n+m}=b_{m} \\
& x_{i} \geqslant 0 \quad i=1(1) n+m .
\end{array}
$$

$$
a_{1 n+1}=\ldots=a_{m n+1}=-1
$$

and all $c_{j} j=1$ (1) $n$ be non-negative so that, in the first tableau, the first $n$ elements in the bottom row are nonpositive (since we minimize). We call such a tableau dual feasible. If, in addition, all $b_{i} i=1$ (1) $m$ are nonnegative then the result is reached. Otherwise apply dual simplex method.

The method

Step 1 (pivot selection). Let $b_{i_{0}}$ be negative. Consider, for all $a_{i_{0}}<0$, $\left|c_{j}\right| a_{i_{0} j} \mid$ and take a smallest. If this is obtained for $j_{0}$ then $a_{k_{0} j_{0}}$ is the pivot.

Step 2. (next-tableau computation). Same as in the Simplex algorithm (Vajda [15], Chung [2]; Gass [4]) or as in Step 3 of Sac. 5.

Step 3 (termination condition). If the bottom row (i.e., $c_{y}$-row) excluding the last element is nonpositive then the solution is reached-terminate. Otherwise go to step 1 .

\section{Examples}

(i) Obtain a nonnegative integral solution of

$$
\left[\begin{array}{rrrr}
-1 & 5 & 1 & 0 \\
1 & 2 & 0 & 1
\end{array}\right]\left[\begin{array}{l}
x_{1} \\
x_{2} \\
x_{3} \\
x_{4}
\end{array}\right]=\left[\begin{array}{l}
5 \\
8
\end{array}\right]
$$


Restricted tableau 0

Restricted tableau 1

(o) $\quad(0) \quad(o) \quad(0)$

$\begin{array}{rccccccrrrrr} & x_{1} & x_{2} & x_{3} & x_{4} & b & & x_{1} & x_{5} & x_{3} & x_{4} & b \\ \text { (1) } x_{5} & -1 & 5+ & 1 & 0 & 5 & x_{2} & -1 / 5 & 1 / 5 & 1 / 5 & 0 & 1 \\ \text { (1) } x_{6} & 1 & 2 & 0 & 1 & 8 & x_{6} & 7 / 5^{+} & -2 / 5 & -2 / 5 & 1 & 6 \\ & 0 & 7 & 1 & 1 & 13 & & 7 / 5 & -7 / 5 & -2 / 5 & 1 & 6\end{array}$

Restricted tableau 2

$\begin{array}{rrrrcc} & x_{6} & x_{5} & x_{3} & x_{4} & b \\ x_{2} & 1 / 7 & 1 / 7 & 1 / 7 & 1 / 7 & 13 / 7 \\ x_{1} & 5 / 7 & -2 / 7 & -2 / 7 & 5 / 7 & 30 / 7 \\ & -1 & -1 & 0 & 0 & 0\end{array}$

A nonnegative solution is thus $x=\left(\begin{array}{llll}30 / 7 & 13 / 7 & 0 & 0\end{array}\right)^{t}$.

Consider the first row as it contains the largest fraction in the value of the variable $x_{2}$, viz., 6/7. Generate the new row (Gomory constraint) as in the algorithm and append this row. Thus

\section{Restricted tableau 20}

$\begin{array}{cccccc} & x_{6} & x_{5} & x_{3} & x_{4} & b \\ x_{2} & 1 / 7 & 1 / 7 & 1 / 7 & 1 / 7 & 13 / 7 \\ x_{1} & 5 / 7 & -2 / 7 & -2 / 7 & 5 / 7 & 30 / 7 \\ s_{1} & -1 / 7 & -1 / 7 & -1 / 7^{+} & -1 / 7 & -6 / 7 \\ & -1 & -1 & 0 & 0 & 0\end{array}$

Restricted tableau 21

$\begin{array}{rrrrrr} & x_{6} & x_{5} & s_{1} & x_{4} & \mathrm{~b} \\ x_{2} & 0 & 0 & 1 & 0 & 1 \\ x_{1} & 1 & 0 & -2 & 1 & 6 \\ x_{3} & 1 & 1 & -7 & 1 & 6 \\ & -1 & -1 & 0 & 0 & 0\end{array}$

Hence a nonnegative integral solution is $x=\left(\begin{array}{llll}6 & 1 & 6 & 0\end{array}\right)^{t}$.

Note. $\mathrm{x}=\left(\begin{array}{llll}0 & 1 & 0 & 6\end{array}\right)^{t}$ could also be another nonnegative integral solution.

(ii) Degenerate case (redundant equation)

Obtain a nonnegative integral solution (Sen [17]) of

$$
\left[\begin{array}{rrrr}
-1 & 2 & 3 & 3 \\
2 & 5 & 6 & 3 \\
-5 & -8 & -9 & -3
\end{array}\right]\left[\begin{array}{l}
x_{1} \\
x_{2} \\
x_{3} \\
x_{4}
\end{array}\right]=\left[\begin{array}{r}
7 \\
16 \\
-25
\end{array}\right]
$$


(o) $\quad(o) \quad(o) \quad(o)$

$\begin{array}{llllllllll}x_{1} & x_{2} & x_{3} & x_{4} & \mathrm{~b} & x_{1} & x_{2} & x_{5} & x_{4} & \mathrm{~b}\end{array}$

(1) $x_{5} \quad-1 \quad 2 \quad \begin{array}{llllllllll}3^{+} & 3 & 7 & x_{3} & -1 / 3 & 2 / 3 & 1 / 3 & 1 & 7 / 3\end{array}$

(1) $\begin{array}{llllllllllll}x_{6} & 2 & 5 & 6 & 3 & 16 & x_{6} & 4 & 1^{+} & -2 & -3 & 2\end{array}$

(1) $\begin{array}{llllllllllll}x_{7} & 5 & 8 & 9 & 3 & 25 & x_{7} & 8 & 2 & -3 & -6 & 4\end{array}$

$\begin{array}{llllllllll}6 & 15 & 18 & 9 & 48 & 0 & 3 & -6 & -9 & 6\end{array}$

Note. The last equation has been multiplied by -1 to make $b_{3}$ positive.

Restricted tableau 2

$\begin{array}{rrcccc} & x_{1} & x_{6} & x_{5} & x_{4} & \mathrm{~b} \\ x_{3} & -3 & -2 / 3 & 5 / 3 & 3 & 1 \\ x_{2} & 4 & 1 & -2 & -3 & 2 \\ x_{7} & 0 & -2 & 1 & 0 & 0 \\ & -12 & -3 & 0 & 0 & 0\end{array}$

The artiflcial variable $x_{7}$ remains in the basis with a zero value. A nonnegative integral solution is $x=\left(\begin{array}{llll}0 & 2 & 1 & 0\end{array}\right)^{t}$.

(iii) Nonnegative nonintegral solution

Obtain a nonnegative integral solution of

$$
\left[\begin{array}{rrr}
1 & 2 & 1 \\
-4 & -2 & 3
\end{array}\right]\left[\begin{array}{l}
x_{1} \\
x_{2} \\
x_{3}
\end{array}\right]=\left[\begin{array}{l}
1 \\
2
\end{array}\right]
$$

Restricted tableau 0

Restricted tableau 1

(o) (o) (o)

$\begin{array}{llllllll}x_{1} & x_{2} & x_{3} & \mathrm{~b} & x_{1} & x_{2} & x_{5} & \mathrm{~b}\end{array}$

$\begin{array}{lrrllllrrrr}\text { (1) } x_{4} & 1 & 2 & 1 & 1 & x_{4} & 7 / 3 & 8 / 3^{+} & -1 / 3 & 1 / 3 \\ \text { (1) } x_{5} & -4 & -2 & 3^{+} & 2 & x_{3} & -4 / 3 & -2 / 3 & 1 / 3 & 2 / 3 \\ & -3 & 0 & 4 & 3 & & 7 / 3 & 8 / 3 & -4 / 3 & 1 / 3\end{array}$

Restricted tableau 2

$\begin{array}{ccccc} & x_{1} & x_{4} & x_{8} & b \\ x_{2} & 7 / 8 & 3 / 8 & -1 / 8 & 1 / 8 \\ x_{3} & -3 / 4 & 2 / 8 & 1 / 4 & 3 / 4 \\ & 0 & -1 & -1 & 0\end{array}$


Hence a nonnegative solution is $\mathrm{x}=\left(\begin{array}{lll}0 & 1 / 8 & 3 / 4\end{array}\right)^{t}$.

Adding the Gomory constraint we have

Restricted tableau 20

$$
\begin{array}{rrrrrrrrrr} 
& x_{1} & x_{4} & x_{5} & b & & s_{1} & x_{4} & x_{5} & b \\
& 7 / 8 & 3 / 8 & -1 / 8 & 1 / 8 & x_{2} & 7 / 2 & -1 / 2 & -1^{+} & -5 / 2 \\
x_{2} & -3 / 4 & 1 / 4 & 1 / 4 & 3 / 4 & x_{3} & -3 & 1 & 1 & 3 \\
x_{1} & -1 / 4^{+} & -1 / 4 & -1 / 4 & -3 / 4 & x_{1} & -4 & 1 & 1 & 3 \\
& 0 & -1 & -1 & 0 & & 0 & -1 & -1 & 0 \\
& & & & & & & & & \\
& s_{1} & x_{4} & x_{2} & b & & & & & \\
x_{5} & -7 / 2 & 1 / 2 & -1 & 5 / 2 & & & & & \\
x_{3} & 1 / 2 & 1 / 2 & 1 & 1 / 2 & & & & & \\
x_{1} & -1 / 2 & 1 / 2 & 1 & 1 / 2 & & & & & \\
& -7 / 2 & -1 / 2 & -1 & 5 / 2 & & & & &
\end{array}
$$

By adding the Gomory constraint we obtain the last row except the last element (viz., 5/2) nonpositive and one artificial variable, viz., $x_{5}$ is still in the basis with the nonzero value $5 / 2$. Hence the equations have no integral solution.

(iv) Inconsistent equations

Obtain a nonnegative integral solution (Sen [11]) of

$$
\left[\begin{array}{lll}
5 & 3 & 2 \\
2 & 1 & 2 \\
4 & 2 & 4
\end{array}\right]\left[\begin{array}{l}
x_{1} \\
x_{2} \\
x_{3}
\end{array}\right]=\left[\begin{array}{r}
10 \\
5 \\
1
\end{array}\right]
$$

\section{Restricted tableau 0}
(o)
(o)
(o)

$\begin{array}{cccccccccc} & x_{1} & x_{2} & x_{3} & b & & x_{1} & x_{6} & x_{3} & b \\ \text { (1) } x_{4} & 5 & 3 & 2 & 10 & x_{4} & -1 & -3 / 2 & -4 & 17 / 2 \\ \text { (1) } x_{5} & 2 & 1 & 2 & 5 & x_{5} & 0 & 1 & 0 & 9 / 2 \\ \text { (1) } x_{6} & 4 & 2^{+} & 4 & 1 & x_{2} & 2 & 1 / 2 & 2 & 1 / 2 \\ & 11 & 6 & 8 & 16 & & -1 & -3 & -4 & 13\end{array}$

The last row except the last element (viz, 13) is nonpositive and two artificial variables, viz. $x_{4}$ and $x_{5}$ are still in the basis with nonzero values. Hence the equations have no nonnegative solution. In fact, the equations have no solution at all. 


\section{Acknowledgement}

The author wishes to thank Dr A A Shamim, Chairman, Computer Centre, Indian Institute of Science for constant encouragement.

\section{References}

[1] Ben-Israel A and Greville T N E 1974 Generalized inverses: Theory and applications (New York: Wiley-Interscience)

[2] Chung A 1966 Linear Programming (Columbus, Ohio: Charles, E. Merrill Books, Inc.)

[3] Farkas J 1901 J.r. Angew. Math. 1241

[4] Gass S I 1975 Linear programming : Methods and applications (New York: McGrawHill)

[5] Gomory R 1963 'All-integer integer programming algorithm' in Industrial Scheduling (eds Muth and Thompson) (Englewood Cliffs., New Jersey : Prentice-Hall)

[6] Gomory R 1963 Recent advances in mathematical programming (eds Graves and Wolfe), (New York : McGraw-Hill)

[7] Gottfried B S and Weisman J 1973 Introduction to optimization theory (Englewood Cliffs, New Jersey : Prentice-Hall, Inc.)

[8] Hurt M F and Waid C 1970 SIAM J. Appl. Math. 19547

[9] Marcus M and Minc H 1964 A Survey of matrix theory and matrix inequalities (Boston, Mass. : Allyn and Bacon)

[10] Salkin H M 1975 Integer programming (Reading, Mass. : Addison-Wesley)

[11] Sen S K 1978 J. Indian Inst. Sci. 6141

[12] Sen S K and Shamim A A 1978 J. Indian Inst. Sci. 60111

[13] Strum J E 1972 Introduction to linear programming (San Francisco : Holden-day)

[14] Vajda S 1974 Theory of linear and nonlinear programming (London : Longman)

[15] Vajda S 1975 Problems in linear and nonlinear programming (London : Charles Griffin) 